\title{
FIELD GROUND TRUTHING DATA COLLECTOR - A MOBILE TOOLKIT FOR IMAGE ANALYSIS AND PROCESSING
}

\author{
X. Meng \\ Wuhan University, No. 37, Luoyu Road, Wuhan, Hubei Province 430079, China - xmeng@whu.edu.cn
}

Commission IV, IV/5

KEY WORDS: Mobile Mapping, Web Services, Spatial Query, Image Analysis and Processing, Visualization

\begin{abstract}
:
Field Ground Truthing Data Collector is one of the four key components of the NASA funded ICCaRS project, being developed in Southeast Michigan. The ICCaRS ground truthing toolkit entertains comprehensive functions: 1) Field functions, including determining locations through GPS, gathering and geo-referencing visual data, laying out ground control points for AEROKAT flights, measuring the flight distance and height, and entering observations of land cover (and use) and health conditions of ecosystems and environments in the vicinity of the flight field; 2) Server synchronization functions, such as, downloading study-area maps, aerial photos and satellite images, uploading and synchronizing field-collected data with the distributed databases, calling the geospatial web services on the server side to conduct spatial querying, image analysis and processing, and receiving the processed results in field for near-real-time validation; and 3) Social network communication functions for direct technical assistance and pedagogical support, e.g., having video-conference calls in field with the supporting educators, scientists, and technologists, participating in Webinars, or engaging discussions with other-learning portals. This customized software package is being built on Apple iPhone/iPad and Google Maps/Earth. The technical infrastructures, data models, coupling methods between distributed geospatial data processing and field data collector tools, remote communication interfaces, coding schema, and functional flow charts will be illustrated and explained at the presentation. A pilot case study will be also demonstrated.
\end{abstract}

\section{INTRODUCTION}

The NASA funded ICCaRS (Investigating Climate Change and Remote Sensing) Project, is being developed in Southeast Michigan by Eastern Michigan University and Wayne County Regional Educational Service Agency. The four key components of the project are the NASA AEROKATS TwinCam-AeroPod Field Operation Manual and the Image Processing Lab Guide, ICCaRS NASA STEM Instructional Units, ICCaRS eLearning Collaboratory and the Field Ground Truthing Data Collector. The Collaboratory is an eLearning portal which includes a web-based geographic information mapping system, an field observation database and group-ware for social networking to build the supporting community. The goal of ICCaRS is that K9-12 sudents and teachers will have a working understanding of the science behind global climate change and its relationship to land-use and land-cover (LULC) changes on multiple scales through NASA data products and models. Students and teachers will learn how remotely sensed data can be used to study the phenomena of global climate change on multiple levels (i.e., scales of size: local, regional and global), be able to acquire remotely sensed data and produce meaningful information (including vegetation index, biomass and LULC change over time) from that data. The Field Ground Truthing Data Collector is focussed on the purpose, through serving as field communication portal to transmit field data to server, and as mobile toolkit for image analysis and processing.

Since providing current mobile phones with more sensing capabilities would greatly enhance the humans presence classification accuracy given the broader input to the classifiers feature vectors (Miluzzo, 2008), the developing
ICCaRS ground truthing toolkit entertains comprehensive functions, including Field Function, Server Synchronization Functions and Social Network Communication Functions. In this study, the customized software package is being built on Apple iPhone/iPad and Google Maps/Earth.

The remainder of the paper is organized as follows: Section 2 gives the comparison of the current top-end handheld mobile phones to illustrate the reason for choosing iPhone/iPad as the handheld platform, while Section 3 describes the technical infrastructures of the ICCaRS eLearning Collaboratory web-portal as well as the handheld ground truthing data collector. Section 4 gives the four primary data models in the ICCaRS project. Section 5 and 6 describe the remote communication interfaces and the coupling methods between image analysis and field data collector tools. In Section 7, the comprehensive functions of the case study are demonstrated. Finally, Section 8 concludes the paper and discusses future work.

\section{COMPARISON OF TOP-END HANDHELD MOBILE PHONES}

In what follows, we present a short qualitative comparison of the Google Nexus One, Nokia N900, Apple iPhone 4 and iPad 2. All these devices are actively being used in support of mobile sensing applications and systems development. The Google Nexus One is the flagship Android device launched by Google and HTC, while the Nokia N900 is currently one of the top-end Nokia mobile phones. Both mobile operating systems, Android and Maemo, are open source software, and can be liberally extended to incorporate new cutting edge technologies as they emerge. However, there's no doubt that 
in the world of technological devices, 2011 was a great year for Apple's products, the iPhone and iPad.

A simple comparison of some of the technical details of the four handheld devices is reported in Table 1. As shown in Table 1, all four platforms present similar computational capabilities given similar processors, large storage and memory size. The screen size of iPad is much bigger than the Nexus One, N900 and iPhone, which is much better for "expressing the software" (Bosker, 2010), especially for expressing mobile mapping applications, photos and images. However, the 9.7 inch screen makes the weight of iPad heavier than the other three. The iPad also has cameras at front and backside. Since taking photos are very important for field data collection, built-in cameras become one of the people-centric features.

Both iPhone and iPad run the same operating system, iOS, originally developed for the iPhone. The specialties of the development of iPhone/iPad applications are illustrated in the next section. Because the operating systems are the same, all iPhone applications are compatible with iPad. The mobile toolkits developed for ICCaRS are for both handheld devices.

Broadcom BCM4750UBG, which iPhone and iPad use as the core chipset, is a single-chip GPS solution. This chipsets is designed for Assisted GPS (A-GPS or aGPS) solution which can reduce the time-to-first-fix (TTFF) and possibly increase the sensitivity even further (Jarvinen 2002). Normally, standalone GPS must search for satellite signals in a clear view of the sky, but A-GPS additionally uses network resources to locate and utilize the satellites faster even when the receiver is located in poor signal conditions.

Besides A-GPS, the common technologies which can be used for receiving location information in the Field Ground Truthing Data Collector are:
Cellular: All cell tower locations are known precisely, so if one handheld mobile is communicating with either GSM or 3G, the mobile can pinpoint location to roughly 1500 meters. Normally, several towers can be detected, so the first rough location can be obtained and calculated in 10 seconds or less. This calculated data is most times less precise than waiting for satellite data.

WiFi: WiFi data are used to obtain position. Although not as accurate as GPS technology, indoor locations can be obtained additionally to outdoor positions. Apple built a database, leveraging its large base of users to log basic WiFi data. The technology used here is called WiFi Positioning System (WPS). The database stores the MAC of the WiFi access point you are connected to and links this MAC to a location. Most WiFi signals work within 20 meters (with walls) to 200 meters (no obstacles), but the accuracy also dependent on whether the location was entered into the database correctly.

Digital Compass: It provides directional information that GPS can't. Digital compass is also helpful for coupling the local scale measurements with landscape scale remote sensing data such as satellite or the high altitude aerial photography taken by NASA AEROKATS TwinCam-AeroPod.

Because the technologies and models are different, the performance of these "high sensitivity" GPS (HSGPS) receivers in terms of TTFF and accuracy does vary [5]. Many efforts have been reported in the literature on evaluation of handheld devices (Miluzzo, 2008, Thiagarajan, 2010, Zhang, 2010). It is generalized that all those GPS receivers performed well in urban-like environment or indoors, and the computational capability of the iPhone/iPad is sufficient to handle high load fast fourier transform (FFT) calculations. Since the iPhone/iPad can provide a good position, velocity and time (PVT) solution, the customized software packages of ICCaRS project are built on them.

\begin{tabular}{|c|c|c|c|c|c|c|c|c|}
\hline Device & $\begin{array}{l}\text { Release } \\
\text { Year }\end{array}$ & $\begin{array}{l}\text { Operating } \\
\text { System }\end{array}$ & CPU & Memory & Storage & Display & Camera & Weight \\
\hline $\begin{array}{l}\text { Google } \\
\text { Nexus } \\
\text { One }\end{array}$ & 2010 & Android 2 & $\begin{array}{l}1 \mathrm{GHz} \\
\text { Qualcomm } \\
\text { QSD 8250 } \\
\text { Snapdragon }\end{array}$ & $\begin{array}{l}512 \mathrm{MB} \\
\text { DRAM }\end{array}$ & $\begin{array}{l}512 \mathrm{MB} \\
\text { Flash } \\
\text { memory, } \\
\text { microSD, } \\
\text { expandable } \\
\text { up to } 32 \mathrm{~GB}\end{array}$ & $\begin{array}{l}3.7 \text { in (9.4 } \\
\text { cm) diagonal, } \\
480 \times 800 \text { px }\end{array}$ & $\begin{array}{l}5.0 \mathrm{MP} \\
\text { Backside }\end{array}$ & $\begin{array}{l}0.29 \mathrm{lb} \\
(130 \mathrm{~g})\end{array}$ \\
\hline $\begin{array}{l}\text { Nokia } \\
\text { N900 }\end{array}$ & 2009 & Maemo 5 & $\begin{array}{l}600 \mathrm{MHz} \\
\text { TI OMAP } \\
3430\end{array}$ & $\begin{array}{l}256 \mathrm{MB} \\
\text { Mobile } \\
\text { DDR, } \\
768 \mathrm{MB} \\
\text { swap space }\end{array}$ & $\begin{array}{l}256 \text { MB } \\
\text { NAND flash, } \\
32 \text { GB eMMC } \\
\text { flash }\end{array}$ & $\begin{array}{l}3.5 \text { in (8.9 } \\
\text { cm) diagonal, } \\
800 \times 480 \mathrm{px}\end{array}$ & $\begin{array}{l}5.0 \mathrm{MP} \\
\text { Backside } \\
0.3 \mathrm{MP} \\
\text { Front }\end{array}$ & $\begin{array}{l}0.4 \mathrm{lb} \\
(181 \mathrm{~g})\end{array}$ \\
\hline $\begin{array}{l}\text { Apple } \\
\text { iPhone } 4\end{array}$ & 2010 & iOS 4 & $\begin{array}{l}1 \mathrm{GHz} \\
\text { Apple A4 }\end{array}$ & $\begin{array}{l}512 \mathrm{MB} \\
\text { eDRAM }\end{array}$ & $\begin{array}{l}16 \text { or } \\
32 \text { GB, } \\
\text { Flash memory }\end{array}$ & $\begin{array}{l}3.5 \text { in ( } 8.9 \\
\mathrm{~cm}) \text { diagonal, } \\
960 \times 640 \mathrm{px}\end{array}$ & $\begin{array}{l}\text { 5.0 MP } \\
\text { Backside } \\
3.0 \mathrm{MP} \\
\text { Front } \\
\end{array}$ & $\begin{array}{l}0.3 \mathrm{lb} \\
(137 \mathrm{~g})\end{array}$ \\
\hline $\begin{array}{l}\text { Apple } \\
\text { iPad } 2\end{array}$ & 2011 & iOS 4 & $\begin{array}{l}1 \mathrm{GHz} \\
\text { Apple A4 }\end{array}$ & $\begin{array}{l}256 \mathrm{MB} \\
\text { DRAM }\end{array}$ & $\begin{array}{l}16,32, \text { or } \\
64 \mathrm{~GB}, \\
\text { Flash memory }\end{array}$ & $\begin{array}{l}9.7 \text { in }(25 \mathrm{~cm}) \\
\text { diagonal, } \\
1024 \times \\
768 \mathrm{px} \\
\end{array}$ & $\begin{array}{l}5.0 \mathrm{MP} \\
\text { Backside } \\
3.0 \mathrm{MP} \\
\text { Front } \\
\end{array}$ & $\begin{array}{l}1.6 \mathrm{lb} \\
(730 \mathrm{~g})\end{array}$ \\
\hline
\end{tabular}

Table 1 Platform Comparison for the Google Nexus One, Nokia N900 and Apple iPhone/iPad 


\section{TECHNICAL INFRASTRUCTURES}

Figure 1 illustrates the technical infrastructures of the web-portal (eLearning Collaboratory) as well as handheld device (Field Ground Truthing Data Collector). The current technical infrastructures build on the preliminary work, also by Eastern Michigan University and Wayne County Regional Educational Service Agency (RESA), who developed a web-based GIS/database in the RESA H2OMapper which is piloted as part of a watershed program targeting middle and early high school learners (Meng, 2009).

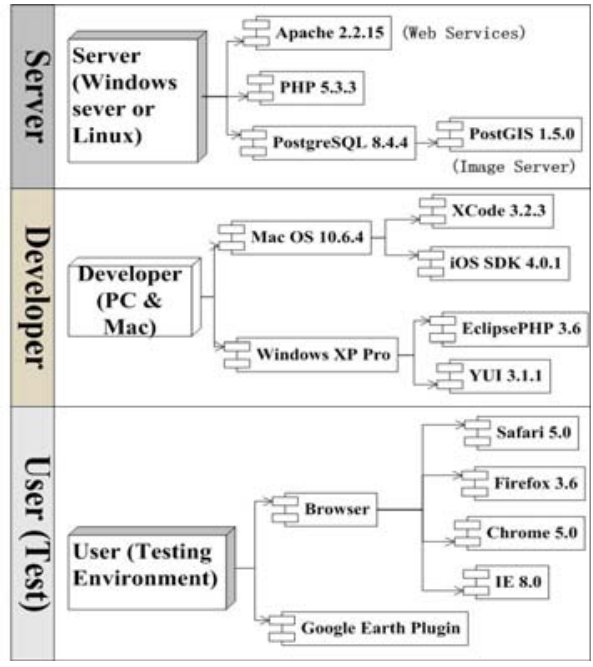

Figure 1. Technical Infrastructures of Web-portal as well as the Handheld

On the server side, the Apache HTTP Server are installed and configured for providing web services including geospatial information services. The server holds PostgreSQL as the database integrated with PostGIS which supports spatial data management, and save the images. The Collaboratory uses PHP as scripting language to develop the Web 2.0 sites.

On the developer side, EclipsePHP and YUI are two useful open source tools for PHP development. The package of Xcode and iOS SDK can be downloaded from Apple website, and includes the Xcode IDE, iOS Simulator, and a suite of additional tools for developing apps for iPhone and iPad.

On the user (test) side, the eLearning Collaboratory works best with the browsers which support Web 2.0, such as Chrome 6+, Firefox $3.6+$, Safari $5^{+}$or Opera $10^{+}$. The Google Earth plug-in allows users to navigate and explore geographic data on a 3D globe using a web browser through retrieving the geospatial data from web services. However, so far, it cannot work with Apple handheld devices. In order to make the web-based GIS of eLearning Collaboratory working, a toolkit for converting Google Earth plug-in maps to Google Maps which can work with iPhone/iPad is also being developed.

The iPhone/iPad applications are written in Objective-C. There are yearly costs to be registered to develop on the platform and publish the application. Not only that, developers can only use the Apple-defined public APIs. The iPhone/iPad indeed has drawbacks but still has excellent support for HTML5 web applications with its Safari browser using WebKit engine. This specialty is good for realizing a fast web-based mobile mapping. It should be also noted that iOS 4 does not fully support multitasking. It supports background threads for tasks such as music playback and network polling. All other application threads are frozen when the application is deactivated (Dorokhova, 2009).

\section{DATA MODELS}

Following are four primary data models in the ICCaRS project. The four models are divided according to functional needs, but mutual overlapping.

1. User-Roles Data Model: representing the user-roles relationship

2. Platform Routine Data Model: representing the routine functions of eLearning Collaboratory and Field Ground Truthing Data Collector

3. Geospatial Data Model: representing the geospatial information retrieved through web services

4. Ground Truthing Data Model: representing the combination of various data collections

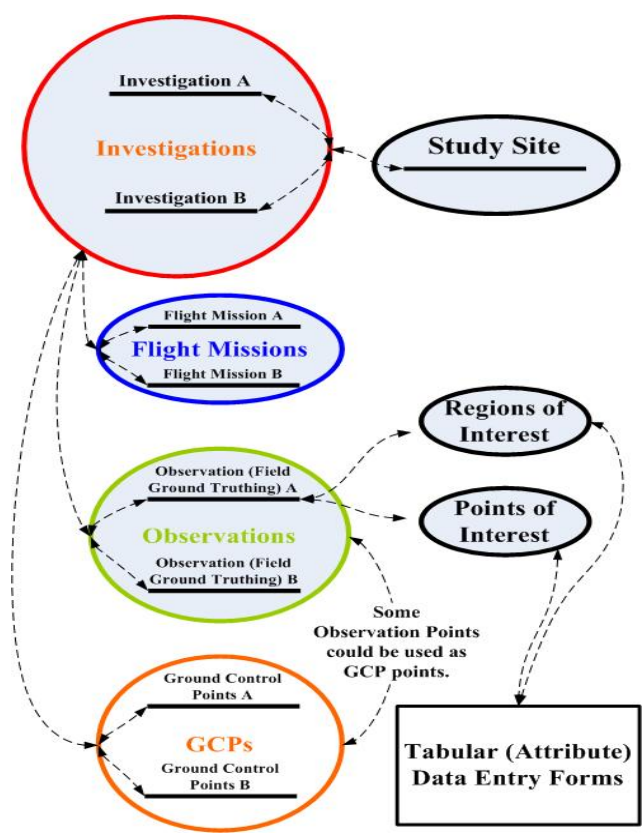

Figure 2. Ground Truthing Data Model

Figure 2 shows the relationship among key data entities of Ground Truthing Data Model. For instance, School A does an investigation, which name is Investigation A, at a Study Site. School A will get the flight mission data by means of flying the NASA AEROKATS TwinCam-AeroPod. Also School A will do some observations in this area. Some observation point could be used as ground control point (GCP). Besides the observation points, some identifiable point could be also used as GCPs. Tabular data entry forms on different areas, such as clouds, soils, temperatures and etc., are prepared for field data collection at the points and regions of interest. 


\section{REMOTE COMMUNICATION INTERFACES}

From the early mobile devices which always only supported GSM interfaces, to the current iPhone and Andriod ranges that incorporate numerous connectivity interfaces such as 3G, WiFi, Bluetooth and GPS, handheld devices become much more powerful. WiFi or 3G connectivity enables developers to create intuitive and function rich mobile applications capable of connecting to external services hosted around the world. Coupling GPS with WiFi and 3G enabled devices yield an even greater potential for integration and innovation.

The Field Ground Truthing Data Collector is a proposed collaboration toolkit that is created by a framework of components utilizing GPS, Internet connectivity and web services. It communicates in sync to provide client users with a set of platform and device independent tracking and collaboration features, which are possible by exploiting the interfaces available to the device. If a particular user is connected to a WiFi hotspot, the mobile device operating system is smart enough to determine the best way of connecting to the content requested by the user. If WiFi connectivity is not available, the device attempts to fetch the same content by other means of communication, usually initiating a 3G data connection (Savic 2010).

Web services for data synchronizing, geo-position, spatial query, image analysis and processing are defined in an XML format that describes the network services as set of endpoints or ports. In WSDL, the abstract definition of endpoints and messages (functions and functions parameters) are separated from their concrete network deployment or data format bindings. The core components of the mobile data collector toolkit are the web services defined by the WSDL definition. Further looking into the "updatePosition" operation which is for geo-position, a detailed code definition is defined below:

\$webServer $=$ new soap_server();

\$namespace= 'http://geodata.acad.emich.edu/iccars

/mobiletoolkit/services/ groundTruthing.php?wsdl';

\$webServer->configureWSDL('groundTruthingServices');

$\$$ webServer $->$ wsdl->schemaTargetNamespace $=$ \$namespace; \$soapAction $=$ false;

\$style = 'rpc';

\$use = 'encoded';

\$methodName = 'updatePosition';

\$description = 'Update position method. Automatically called when longatude and latitude position is updated.';

\$input = array('longitude' => 'xsd:string','latitude' $=>$ 'xsd:string', 'token' => 'xsd:string','trackid' => 'xsd:int');

\$output = array('return' => 'xsd:string');

\$webServer->register(\$methodName, \$input, \$output, \$namespace, \$soapAction, \$style, \$use, \$description);

\section{COUPLING METHODS BETWEEN IMAGE ANALYSIS AND FIELD DATA COLLECTOR TOOLS}

Spatial analysis (or process) which includes spatial query, feature and image analysis using the technology of distributed geospatial database systems becomes more and more important because of its capacity of supporting data interoperability in transmission (Ramirez 2001).
There exists a need for publishing, sharing, and accessing spatial data, which are AEROKATS TwinCam-AeroPod images, from distributed geospatial databases interconnected through web-based geo-information services. The technical problems can be induced to three main aspects: (1) Finding localization of the required images, (2) Enabling remote access to the images, and (3) Transforming different image representations. In order to solve the technical challenges, the conformance to standards is essential for distributed geospatial database systems. OGC Web Processing Service standards and use of web services chaining (Meng 2010) could offer the solution for the above issues, and make the distributed image analysis and processing feasible.
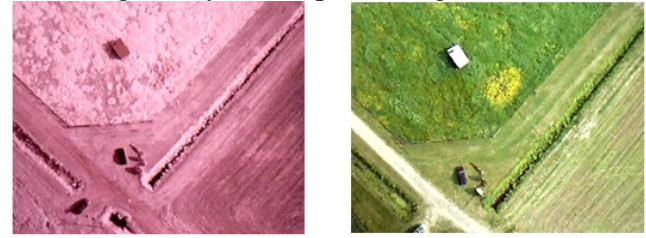

The images in the same view field

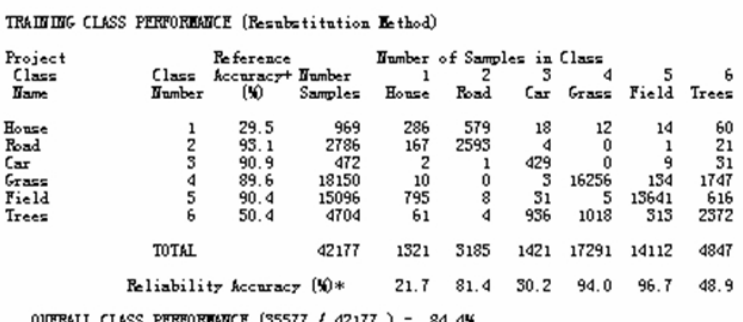

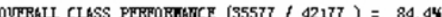

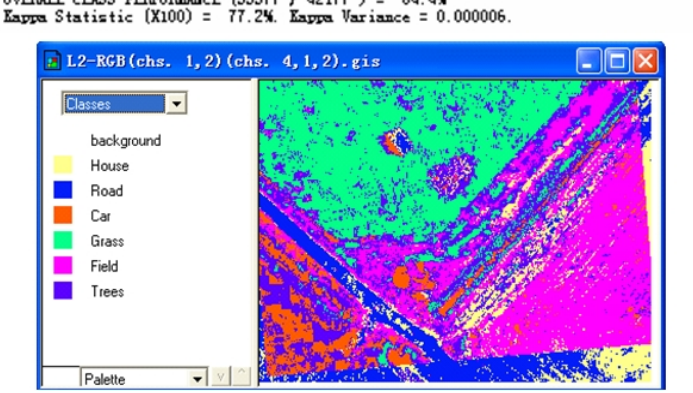

Figure 3. The images in the same view field, and the processed result which is conducted by MultiSpec

By coupling digital aerial and ground photos, A-GPS, field data collected with the mobile devices and web services applied in the eLearning Collaboratory server enabled rapid sampling and recording geographic position (latitude/longitude) of investigation site with vegetation, watershed and soil parameters. In other words, coupling our local scale measurements with landscape scale remote sensing data such as satellite and the high altitude aerial photography which is taken by the NASA AEROKATS TwinCam-AeroPod, we can have a complete picture of land-use and land-cover changes on multiple scales.

Remote sensing data, in conjunction with image analysis software, are being used to quantify vegetation cover (Louhaichi, 2010). Recently, we have two cameras: one for near infrared (NIR) video and the other for visible bands video. We are searching for good quality of paired images: one NIR image matching with another visible-band image in 
terms of exactly the same view field. After exporting the video files taken by the NASA AEROKATS TwinCamAeroPod, a large number of images are generated. But only a small percentage of them have good quality and can be used to find "matching pairs." Figure 3 shows the images in the same view field, and the processed result which is conducted by MultiSpec. The "Training Class Performance" table tabulates how well the pixels of each field and the classes were classified. If the Reference Accuracy is particularly low (say less than $50 \%$ ) for a class, the training pixels for that class should be reexamined and new training pixels should be selected. The higher of the Overall Class Performance, the better of the classification result.

\section{GROUND TRUTHING TOOLKIT FUNCTIONS}

The ICCaRS field ground truthing toolkit entertains comprehensive functions, such as field functions, server synchronization functions and social network communication functions, for image analysis and processing.

\subsection{Field Functions}

The ICCaRS ground truthing toolkit is a kind of GIS visualization. It has graphical software interface which makes it easy to learn and simple operated. It is being developed to provide various position data and attribute information collection methods, such as manual collection, automatic collection, public point collection, offset collection, manual inputting and menu selection. Figure 4 represents the user interface of the ICCaRS field ground truthing data collector. Furthermore, internal microphone is used for real time voice information for marking attributes. Field functions of the ICCaRS ground truthing toolkit include:

1. Determining locations through A-GPS which was illustrated in Section 2

2. Gathering and geo-referencing audio and visual data

3. Laying out ground control points for AEROKAT flights

4. Measuring the flight distance and height

5. Entering observations of land cover (and use) and health conditions of ecosystems and environments in the vicinity of the flight field

\subsection{Server Synchronization Functions}

The handheld device is able to real time update data to server on study site, and achieves real time management through web services which are deployed in the Apache server side. Server synchronization functions of the ICCaRS ground truthing toolkit include:

1. Uploading and synchronizing field-collected data with the main database on the server

2. Calling the tools on the server to conduct image analysis and processing

3. Receiving the image-processed results in field for near-real-time validation

4. Downloading study-area maps, aerial photos and satellite images

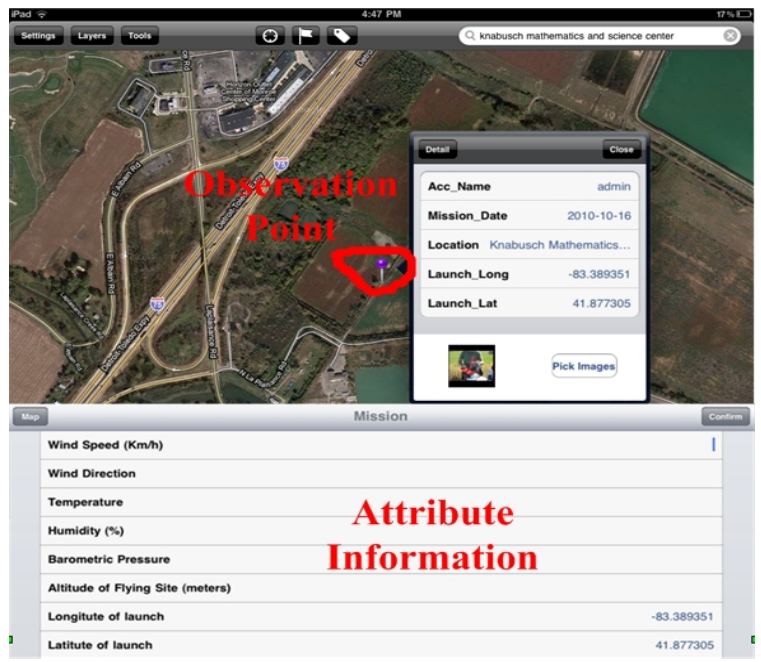

Figure 4. User Interface of the ICCaRS Field Ground Truthing Data Collector

Figure 5 illustrates the functional flow chart of server synchronization and related web services. The authorized clients who hold the iPhone/iPad have the ability to submit the data to the server side through "Data Submitting Service" and "Image Submitting Service". The Client also needs to call the Server for retrieving the image-processed results through "Image Analysis Retrieving Service". There is a stack used as "Download Warehouse" storing the status of the image as well as the attribute forms, and the status shows whether the images are ready for downloading or not. "Status Checking Service” is used for checking the "ready" status.

\subsection{Social Network Communication Functions for Direct Technical Assistance and Pedagogical Support}

Since the ICCaRS field ground truthing data collector uses the iPhone/iPad platform, it can provide users the original functions, like browsing the web, enjoying photos and videos, books, periodicals and music, taking notes, emailing, doing social networking, participating in Webinars, and engaging discussions with other-learning portals. Thousands of Apple applications can be found from the App store. Almost all current social networking applications have launched an iPhone and/or iPad application.

The ICCaRS project is coupling with social networks, Facebook, Twitter, Google Picasa and Blogger. These social applications are embedded into a user's workspace (as shown in Figure 6) of the Collaboratory portal. Users can use their workspaces when they are doing field observations, if they have 3G connection. Video-conference calls in field with the supporting educators, scientists, and technologists, participating in Webinars, or engaging discussions with other-leaning portals. Users can also use the iPhone/iPad to have video calls, with the assistance of a FaceTime application, to another iPhone/iPad.

\section{CONCLUSIONS}

This paper outlines how to couple handheld devices, geo-referenced AEROKATS TwinCam AeroPod photos and 
web services in order to achieve image analysis and processing. A mobile toolkit is developed for the field ground truthing data collector. A prototype web platform for deploying the services is also developed in the ICCaRS eLearning Collaboratory portal. The future work of our study will concentrate on realizing more sophisticated distributed geoinformation services for image analyses and processing, particularly on the remote accessing and manipulation of large geo-referenced images. Some applications, like using Normalized Difference Vegetation Indices (NDVI) data to compute biomass and predict yield, will be considered to be put into the mobile toolkit.

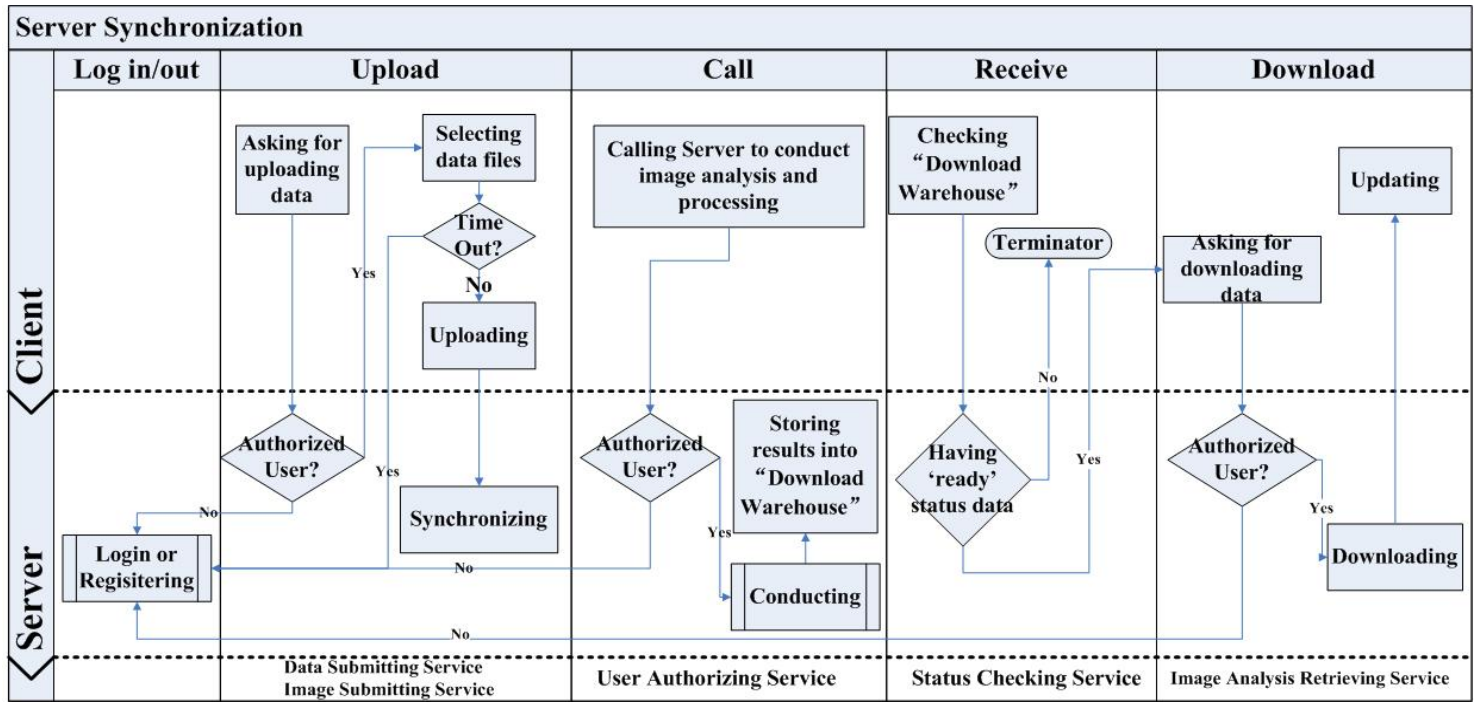

Figure 5. Functional Flow Chart of Server Synchronization

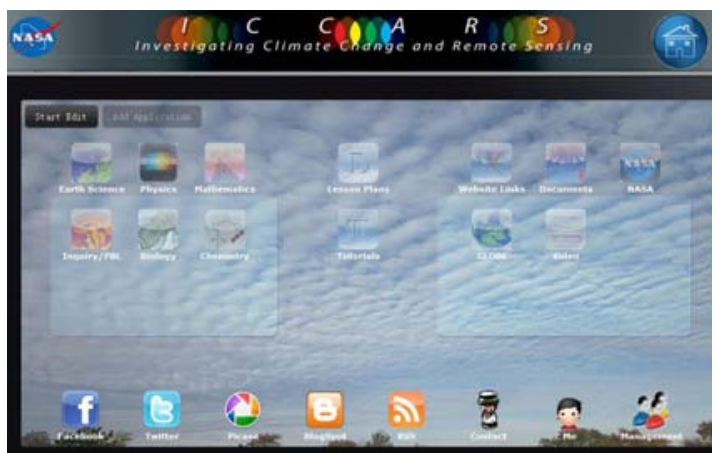

Figure 6. User’s Workspace

\section{REFERENCES}

Bosker, B., 2010. “Apple's iPad 2 Won't Be A Smaller, 7-Inch Version, Steve Jobs Suggests”. The Huffington Post. http://www.huffingtonpost.com/2010/10/19/apples-ipad-2-wo nt-be-a-s_n_767882.html (7 Jan. 2011).

Dorokhova, R., Amelichev, N., Krinkin, K., 2009. "Evaluation of Modern Mobile Platforms from the Developer Standpoint”.http://osll.spb.ru/attachments/download/427/eval uation_edit_1.doc (11 Jan. 2011).

Jarvinen, J., DeSalas, J., LaMance, J., 2002. “Assisted GPS: A Low-Infrastructure Approach”. GPS World. http://www.gpsworld.com/gps/assisted-gps-a-low-infrastruct ure-approach-734 (11 Jun. 2008).

Louhaichi, M., Johnson, M., Woerz, A., Jasra, A., and Johnson, D., 2010. Digital charting technique for monitoring rangeland vegetation cover at local scale. International Journal of Agriculture \& Biology, 12, pp. 406-410.

Meng, X., Bian, F., Xie, Y., 2009. The Application of Web-based GIS and GPS Technology to Assess Water Quality in Michigan. In: The 3rd International Conference on Intelligent Information Technology Application, Volume 1, pp. 517-520.

Meng, X., Xie, Y., Bian, F., 2010. Distributed Geospatial Analysis through Web Processing Service: A Case Study of Earthquake Disaster Assessment. Journal of Software, 5(6), pp. 671-679.

Miluzzo, E., Oakley, J., Lu, H., Lane N., Peterson, R., Campbell, A., 2008, Evaluating the iPhone as a mobile platform for people-centric sensing applications. In Proc. of Intl Workshop on Urban, Community, and Social Applications of Networked Sensing Systems (UrbanSense08). Raleigh, NC, USA, pp. 41-45.

Ramirez, M., 2001. Distributed Processing of Spatial Queries. Tese Dsc, COPPE/UFRJ

Savic, S., Shi, H., 2010. TEAMTRACKER - An Innovative Team Collaboration System. International Journal of Computer Networks \& Communications (IJCNC) 2(5), pp. 105-118

Thiagarajan, A., Biagioni, J., Gerlich, T., Eriksson, J., 2010. Cooperative Transit Tracking using Smart-phones. In: SenSys '10 Proceedings of the 8th ACM Conference on Embedded Networked Sensor Systems.

Zhang, J., Binghao Li, B., Dempster, A., Rizos, C., 2010. Evaluation of High Sensitivity GPS Receivers. International Symposium on GPS/GNSS Taipei, Taiwan, pp. 1-6 